\title{
Quantum Monte Carlo calculations of molecular electron affinities: First-row hydrides
}

\author{
Gabriele Morosia) and Massimo Mellab) \\ Dipartimento di Chimica Fisica ed Elettrochimica, Università di Milano, via Golgi 19, 20133 Milano, Italy \\ Dario Bressaninic) \\ Dipartimento di Scienze Chimiche, Fisiche e Matematiche, Università dell' Insubria, Polo di Como, \\ via Lucini 3, 22100 Como, Italy
}

(Received 7 June 1999; accepted 27 July 1999)

\begin{abstract}
Very accurate energies can be computed by the fixed-node diffusion Monte Carlo method. They are affected only by the nodal error due to the approximate description of the nodal surfaces by the trial wave function. We examine the cancellation of nodal errors in molecular electron affinity calculations. Ground state energies of the anions of first-row hydrides $\mathrm{AH}(\mathrm{A}=\mathrm{Li}-\mathrm{O})$ have been computed using the fixed-node diffusion Monte Carlo method with a determinant times a correlation factor as the trial wave function. The energies are among the lowest to date. Using the energy values for the neutral molecules computed by Luchow and Anderson [A. Luchow and J. B. Anderson, J. Chem. Phys. 105, 7573 (1996)] we computed adiabatic electron affinity values and found them in agreement with the experimental data. As a consequence, the values of the anion dissociation energies are also correctly evaluated. (c) 1999 American Institute of Physics.
\end{abstract}

[S0021-9606(99)30339-1]

\section{INTRODUCTION}

The calculation of atomic and molecular electron affinities is one of the most serious problems in quantum chemistry. The electron affinity (EA) may be decomposed into contributions of

$$
\mathrm{EA}=\mathrm{EA}_{\mathrm{HF}}+\mathrm{EA}_{c}+\delta \mathrm{EA},
$$

where $\mathrm{EA}_{\mathrm{HF}}$ is the energy difference between the anion and the neutral at the Hartree-Fock (HF) level, EA is the correlation contribution, and $\delta \mathrm{EA}$ is a small correction for relativistic effects and nuclear motion. $\mathrm{EA}_{\mathrm{HF}}$ can be easily evaluated, but most anions are unbound at this level of theory, so correlation is crucial to evaluate EAs. The correlation energy of the anion is larger than that of the neutral system and the problem is particularly serious when the electron added makes a new electron pair. Getting a balanced description of the two systems is an even more difficult task than calculation of the dissociation energy. Pople et al. ${ }^{1}$ suggested that to circumvent this problem of balance one should compute the EA using isogyric comparisons (ICs) to the hydrogen molecule. By combining the electron attachment process, that results in new electron pair formation, with the hydrogen molecule dissociation reaction, the number of unpaired electrons remains constant and any error in the computed correlation energy is partly cancelled by the corresponding error in the hydrogen dissociation energy. Results for first-row compounds agree with experimental data to within $0.1 \mathrm{eV}$. So accurate prediction of the EA requires the calculation of a percentage of correlation energies as large as possible, oth-

\footnotetext{
${ }^{a}$ Electronic mail: gabriele.morosi@unimi.it

b)Electronic mail: massimo.mella@unimi.it

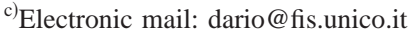

erwise one has to rely on fortuitous cancellations of errors. Traditional $a b$ initio methods introduce the orbital approximation to avoid the evaluation of integrals over three and four electrons, but they include interelectronic distance only implicitly so their convergence towards the exact solution of the Schrödinger equation is very slow. Explicitly correlated wave functions can be calculated by the " $r_{12}$ method" developed by Kutzelnigg and co-workers. ${ }^{2-4} \mathrm{~A}$ linear term in the interelectronic distance allows one to satisfy the cusp condition, but to avoid the calculation of integrals over 9 and 12 dimensions the resolution of the identity is approximated in the one-electron basis set given. Recently this method has been applied to the calculation of electron affinities of firstrow atoms, the maximum deviation from experiment being $-16 \mathrm{meV}$ for $\mathrm{F}^{5}$ Among explicitly correlated functions only correlated Gaussian functions allow analytical integration and they can give very accurate results, provided that careful optimization of the nonlinear parameters is performed. Unfortunately this type of function reproduces the cusp conditions poorly, and this has the unpleasant effect of slowing down the convergence.

With regard to the density functional theory a systematic study of EAs computed by six functionals evidenced a very strong dependence on the quality of the exchange functionals and a tendency to overestimate the EA by a few tenths of an electron volt on average. ${ }^{6}$

A totally different approach to the correlation problem is given by the stochastic methods. In principle the diffusion or the Green's function Monte Carlo methods allow one to sample the exact wave function and thus to compute the exact values of the atomic and molecular ground state ener- 
gies and properties. ${ }^{7-9}$ However implementation of the diffusion Monte Carlo technique is hampered by the so-called sign problem, that is, the need to cope with the antisymmetric property of the wave function for a fermion system. The easiest way to deal with the sign problem is to adopt the fixed node approximation diffusion Monte Carlo (FN-DMC), that is, to confine the diffusion process of the walkers within the nodal surfaces of a trial wave function. The FN-DMC method is variational and again we are confronted with the problem of missing a part, even if only a small percentage, of the correlation energy. The errors in the nodal surfaces of the trial wave functions introduce a bias known as nodal error. These nodal errors are of the order of $10 \mathrm{kcal} \mathrm{mol}^{-1}$ for 10 -electron systems, so to compute a property like the electron affinity we must rely on a significant cancellation of errors to achieve a $1 \mathrm{kcal} \mathrm{mol}^{-1}$ accuracy, that is, chemical accuracy. Luchow and Anderson ${ }^{10}$ computed dissociation energies of first-row hydrides with accuracies of 0.5 $\mathrm{kcal} \mathrm{mol}^{-1}$ or better using near HF limit wave functions for the hydrides and the corresponding atoms, so a significant cancellation of errors was achieved when dealing with the same number of electrons in molecule and dissociation products.

In this article we aim to explore the same strategy as Luchow and Anderson and to check whether cancellation of nodal errors can also be achieved in computing EAs, that is, in computing energy differences of systems with $N$ and $N$ +1 electrons.

Few quantum Monte Carlo (QMC) results on atomic EAs have been published: Barnett et al. ${ }^{11}$ using FN-DMC simulations computed a value of $3.45(11) \mathrm{eV}$ for the electron affinity of fluorine against the experimental result 3.399(3) $\mathrm{eV}$. Moskowitz and Schmidt ${ }^{12}$ optimized correlated wave functions for atomic anions by VMC: their EAs differed on average by $0.07 \mathrm{eV}$ from the experimental results. In our study of the positron affinity, ${ }^{13}$ a problem as strongly dependent on the correlation of particle motions as an EA calculation, we used their wave functions as trial wave functions in FN-DMC calculations. We improved the anion energy values, but no improvement was found in the EA values in spite of the larger percentage of correlation energy recovered at this level of theory.

Experimental determination of EAs is a difficult problem due to the need for anion sources of high enough concentration to allow detection of the products of photodetachment by suitable tunable lasers. So a method that could predict EAs with chemical accuracy would be a great achievement. To verify the performance of QMC methods in molecular EA calculations we carried out calculations on diatomic hydrides $\mathrm{AH}(\mathrm{A}=\mathrm{Li}, \mathrm{Be}, \mathrm{B}, \mathrm{C}, \mathrm{N}$, and $\mathrm{O})$. Because the FH EA is negative, it cannot be determined by a bound-state method.

\section{CALCULATIONS}

The quantum Monte Carlo theoretical foundations and recent developments have been thoroughly covered by Hammond et al., ${ }^{7}$ so in the following we only discuss how we implemented those methods in our simulations.

To compute the adiabatic EAs we assumed as reference values for the energies of the neutral first-row hydrides those published by Luchow and Anderson ${ }^{10}$ and computed only the energies of the corresponding anions by the FN-DMC method. We chose basis sets and trial wave functions very similar to the ones used by Luchow and Anderson ${ }^{10}$ in their calculations of the neutral first-row hydrides; in this way we hoped to more easily achieve the nodal error cancellation.

So, as a trial function we assumed the usual product of determinants for $\alpha$ and $\beta$ electrons multiplied by a positive correlation factor,

$$
\Psi_{T}=\operatorname{det} \Phi^{(\alpha)} \operatorname{det} \Phi^{(\beta)} e^{U} .
$$

As a basis set we adopted the one from Cade and Huo, ${ }^{14}$ but constrained the $\sigma$ and $\pi$ orbitals so that they have the same exponent and dispensed the $f$-type atomic orbitals. We investigated the influence of the inclusion of diffuse functions that at the $a b$ initio level are very important to correctly describe anions: the FN-DMC energy is not modified by the presence of diffuse orbitals. Each Slater type orbital (STO) was expanded into six Gaussians. The molecular orbitals were computed at the self-consistent field (SCF) level: for the open shell anions we tried both restricted open shell HF (ROHF) and unrestricted Hartree-Fock (UHF) wave functions to investigate the differences in nodal surfaces: for $\mathrm{LiH}^{-}$and $\mathrm{BH}^{-}$we got the same DMC energies within one standard deviation, but for $\mathrm{NH}^{-}$the DMC energy is $-55.2177(5)$ hartree at the ROHF level and $-55.2107(2)$ hartree at the UHF level. For BeH Luchow and Anderson ${ }^{10}$ also employed a multiconfiguration (MC) wave function,

$$
\Psi_{T}=\left(\sum_{i}^{n} c_{i} \operatorname{det} \Phi_{i}^{(\alpha)} \operatorname{det} \Phi_{i}^{(\beta)}\right) e^{U},
$$

reducing the nodal error by 5 mhartree. We tried a variety of correlated [MC-SCF, configuration interaction (CI), and spin-coupled valence bond (SC-VB)] wave functions, but the largest lowering of the energy of $\mathrm{BeH}^{-}$, in comparison with the value calculated using the SCF trial wave function, was 3 mhartree. In Sec. III of this article we report results computed using ROHF trial wave functions. For the correlation factor $U$ we used the one defined by Schmidt and Moskowitz, ${ }^{15}$ a sum over nuclei and electron pairs of

$$
U_{a i j}=\sum_{k}^{N_{a}} c_{k a}\left(\bar{r}_{a i}^{1 k a} \bar{r}_{a j}^{m_{k a}}+\bar{r}_{a j}^{1} k_{a i} \bar{r}_{a i}^{m_{k a}}\right) \bar{r}_{i j}^{n_{k a}}
$$

where $\bar{r}=b r /(1+b r)$, a and $i, j$ refer to nuclei and electrons, respectively. For all the systems studied, except $\mathrm{LiH}^{-}$, we included $N_{a}=14$ terms, namely, four electron-electron, six electron-nucleus, and four electron-electron-nucleus terms. For $\mathrm{LiH}^{-}$we included $N_{a}=17$ terms, namely, 4 electronelectron, 3 electron-nucleus, and 10 electron-electronnucleus terms. For $\mathrm{BeH}^{-}, \mathrm{BH}^{-}$, and $\mathrm{CH}^{-}$we assumed the parameters optimized by Moskowitz and Schmidt ${ }^{12}$ for the corresponding atomic anions. Only the correlation factors for $\mathrm{LiH}^{-}, \mathrm{NH}^{-}$, and $\mathrm{OH}^{-}$, both the linear coefficients and the nonlinear parameters, were optimized by minimizing the variance of the local energy by the variational Monte Carlo (VMC) method. ${ }^{16}$ Using these trial wave functions we car- 
TABLE I. Ground state geometries, VMC, and FN-DMC energies for firstrow hydride anions. Numbers in parentheses are one standard deviation in the last digit.

\begin{tabular}{cccr}
\hline \hline & $\begin{array}{c}r_{e} \\
(\AA)^{\mathrm{a}}\end{array}$ & $\begin{array}{c}E_{\mathrm{VMC}} \\
(\text { hartree })\end{array}$ & $\begin{array}{c}E_{\mathrm{FN}-\mathrm{DMC}} \\
(\text { hartree })\end{array}$ \\
\hline $\mathrm{LiH}^{-}$ & 1.667 & $-8.0716(3)$ & $-8.0829(1)$ \\
$\mathrm{BeH}^{-}$ & 1.426 & $-15.194(1)$ & $-15.2627(2)$ \\
$\mathrm{BH}^{-}$ & 1.269 & $-25.2417(4)$ & $-25.2798(1)$ \\
$\mathrm{CH}^{-}$ & 1.151 & $-38.4673(8)$ & $-38.5087(1)$ \\
$\mathrm{NH}^{-}$ & 1.037 & $-55.080(3)$ & $-55.2177(5)$ \\
$\mathrm{OH}^{-}$ & 0.971 & $-75.695(2)$ & $-75.7874(3)$ \\
\hline \hline
\end{tabular}

${ }^{\text {a Reference } 17 .}$

ried out VMC and FN-DMC calculations on the anions of first-row hydrides. The bond distances were taken from the article by Rosmus and Meyer. ${ }^{17}$

For DMC simulation a time step $\tau=0.001$ hartree $^{-1}$ was adopted and we checked the time step bias associated with this value by performing simulations with shorter time steps: we found that the difference in energy was within the statistical uncertainty.

Calculations were performed at the experimental geometries or at the coupled electron pair approximation (CEPA) geometries. ${ }^{17}$ For $\mathrm{LiH}^{-}$Franck-Condon analyses of the photoelectron spectrum gave a value $r_{c}=1.724 \pm 0.025 \AA,{ }^{18}$ longer than previous theoretical estimates (see Table 15 of Ref. 19 and the recent result by Gutsev, Nooijen, and Bartlett $^{20}$ ). We performed several FN-DMC calculations at different geometries: our minimum is at $1.675 \AA$, in agreement with the best previous calculations, and its energy is lower by $0.00076(7)$ hartree than the value at $1.724 \AA$. All the theoretical predictions converge towards a $r_{c}$ value that is shorter than the one determined by Sarkas et al. ${ }^{18}$

\section{RESULTS AND DISCUSSION}

The total calculated energies for the anionic AH species for the VMC and FN-DMC simulations are listed in Table I. The uncertainties correspond to one standard deviation in the last digit. The adiabatic experimental, FN-DMC, and VMC EAs are reported in Table II: the VMC values are presented only to stress that the DMC method is very effective in recovering the correlation energy and thus in improving the results. Our adiabatic EAs do not include the contribution of relativistic effects and nuclear motion since these corrections would change the EA within one standard deviation. Our values are compared with a selection of results from the literature computed by different methods. We selected only a few studies that reported data on the series of first-row hydrides: many papers that examined just one or a few hydrides presented even better results, but it is difficult to judge the quality of a method if it is not applied to the whole first row.

$\mathrm{LiH}$ and $\mathrm{LiH}^{-}$have been the subject of numerous theoretical studies (see, for example, Ref. 20, and references therein) performed at different levels of theory with Gaussian, Slater, and numerical basis sets. Confronted with so many results, we performed a simulation for the $\mathrm{LiH}^{-}$system using a more accurate trial wave function in comparison with other hydrides including 17 terms in the correlation
TABLE II. Adiabatic electron affinities in (eV) calculated with the FNDMC method compared with experimental and selected theoretical results. Numbers in parentheses are one standard deviation in the last digit.

\begin{tabular}{llllrrrr}
\hline \hline & \multicolumn{1}{c}{ Experiment } & FN-DMC & VMC & PNO-CI $^{\mathrm{a}}$ & CEPA $^{\mathrm{a}}$ & MP4 $^{\mathrm{b}}$ & IC $^{\mathrm{c}}$ \\
\hline $\mathrm{LiH}$ & $0.342(12)^{\mathrm{d}}$ & $0.340(4)$ & $0.70(1)$ & 0.26 & 0.26 & 0.32 & \\
$\mathrm{BeH}$ & $0.7(1)^{\mathrm{e}}$ & $0.601(8)$ & $0.49(4)$ & 0.27 & 0.48 & 0.49 & 0.64 \\
$\mathrm{BH}$ & & $0.11(1)$ & $0.81(5)$ & -0.11 & 0.03 & 0.07 & 0.12 \\
$\mathrm{CH}$ & $1.238(8)^{\mathrm{f}}$ & $1.24(1)$ & $1.15(6)$ & 0.95 & 1.04 & 1.19 & 1.14 \\
$\mathrm{NH}$ & $0.374(4)^{\mathrm{g}}$ & $0.33(2)$ & $2.23(10)$ & -0.25 & 0.01 & 0.18 & 0.29 \\
$\mathrm{OH}$ & $1.827670(21)^{\mathrm{h}}$ & $1.80(2)$ & $1.28(8)$ & 1.27 & 1.51 & 1.79 & 1.88 \\
\hline \hline
\end{tabular}

${ }^{\mathrm{a}}$ Reference 17, pseudo-natural orbital-CI.

${ }^{\mathrm{b}}$ Reference 21 .

${ }^{\mathrm{c}}$ Reference 1.

${ }^{\mathrm{d}}$ Reference 18 .

e Reference 22.

${ }^{\mathrm{f}}$ Reference 23.

${ }^{g}$ Reference 24.

${ }^{\text {h}}$ Reference 25 .

factor. We also recomputed the neutral: our values are -8.0704(1) hartree for $\mathrm{LiH}$ and $-8.0829(1)$ hartree for $\mathrm{LiH}^{-}$. The adiabatic EA is $0.340(4) \mathrm{eV}$, a value larger than the previous predictions but in the best agreement with the experimental measurements of $0.342 \pm 0.012 \mathrm{eV}$ for ${ }^{7} \mathrm{LiH}$ and $0.337 \pm 0.012 \mathrm{eV}$ for ${ }^{7} \mathrm{LiD} .{ }^{18}$ For $\mathrm{BeH}$ it is difficult to evaluate the precision of our EA because of the large experimental error bar $0.1 \mathrm{eV}$, at least one order of magnitude larger than for the other hydrides; beyond our EA of $0.601(8)$ $\mathrm{eV}$, only the value calculated by isogyric comparison is in agreement with the experiment. Previous results gave lower EAs, giving evidence of the difficulty of correctly describing the correlation of the anion. We met with a similar problem for the nodal error that we were unable to reduce in spite of the use of multiconfiguration trial wave functions.

Our EA value of $0.11(1) \mathrm{eV}$ for $\mathrm{BH}$ suggests that $\mathrm{BH}^{-}$ should be stable; this prediction is in qualitative agreement with most previous calculations, even if our value is slightly larger. The EA of $\mathrm{CH}$ and $\mathrm{OH}$ nearly match the experimental value, but the EA of $\mathrm{NH}$ is within two standard deviations of the experimental value. This is our worst result for the series of first-row hydrides; however previous calculations showed a much larger error.

Our results compare very favorably with previous investigations. To stress the improvement with respect to previous calculations, the differences between experimental and calculated adiabatic EAs of first-row hydrides are plotted in Fig. 1. For $\mathrm{BH}$ there is no experimental value, so to also include $\mathrm{BH}$ in the plot we arbitrarily assigned it our calculated EA of $0.11(1) \mathrm{eV}$. The large bar associated with $\mathrm{BeH}$ is due to the large uncertainty $(0.1 \mathrm{eV})$ of the experimental value. A clear feature of this plot is that the theoretical values underestimate the experimental EAs, that is, the quality of the wave functions of the anions is poorer than that of the neutral systems. Also the DMC values seem to follow this trend even if in general the calculated value is within standard deviation of the experimental result. There is also a trend to increase the difference between the experimental and calculated adiabatic EA when going from $\mathrm{LiH}$ to $\mathrm{OH}$, neglecting $\mathrm{BeH}$. The more electrons the system includes, the lower the percentage of correlation energy that is recovered, and an 


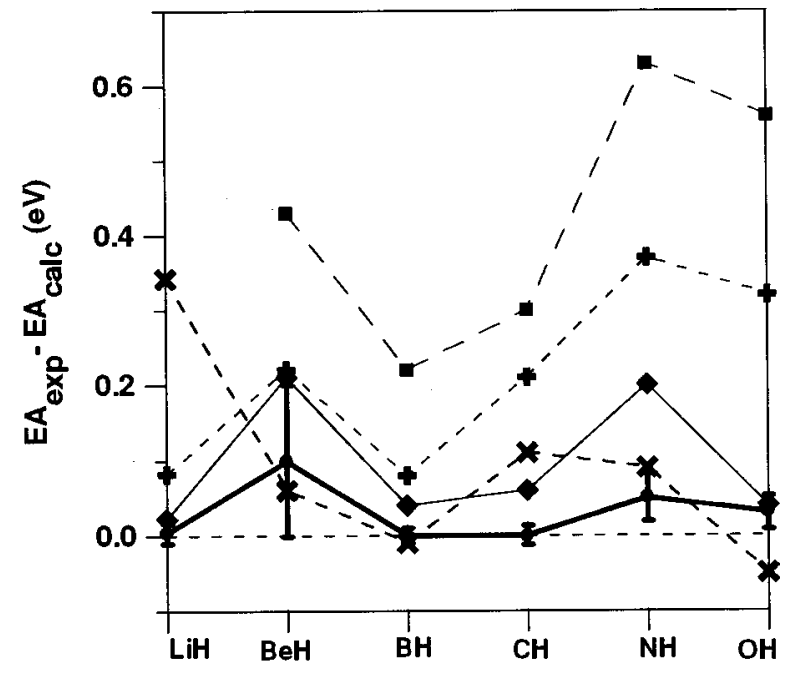

FIG. 1. Difference between the experimental and calculated adiabatic electron affinities of first-row hydrides: FN-DMC $(\mathbf{O})$; PNO-CI (Ref. 17) (ם); CEPA (Ref. 17) (+); MP4 (Ref. 21) ( ); IC (Ref. 1) (×).

accurate balance of the correlation energy in the neutral and in the anion becomes more and more difficult to achieve.

From the FN-DMC energies of the molecular anions reported in Table I and the FN-DMC energies of the neutral atoms ${ }^{10}$ and of the atomic anions ${ }^{13}$ we computed the dissociation energies $D_{c}$ reported in Table III. They correspond to the dissociation reactions of the $\mathrm{AH}^{-}$anions to the neutral atoms $\mathrm{A}$ and $\mathrm{H}^{-}(\mathrm{A}=\mathrm{Li}, \mathrm{Be}, \mathrm{B}$, and $\mathrm{N})$ or $\mathrm{A}^{-}$and $\mathrm{H}(\mathrm{A}=\mathrm{C}$ and $\mathrm{O}$ ). The experimental values $D_{0}$ were taken from the article by Rosmus and Meyer, ${ }^{17}$ except for $\mathrm{LiH}^{-}$whose value has only recently been measured. ${ }^{18}$ The zero point energy corrections were computed either from the experimental data whenever available or from CEPA results; ${ }^{17}$ for $\mathrm{LiH}^{-}$ we adopted the correction calculated by Gutsev et al. ${ }^{20}$ Our values are within one standard deviation of the experimental data; only $\mathrm{OH}^{-}$lies within two standard deviations. However the rather large experimental uncertainty should be reduced to allow a more stringent test of our calculations. As to previous calculations our results are better than previous CEPA values, ${ }^{17}$ while are only a slightly improvement over the Moller-Plesset fourth order perturbation theory (MP4) values. $^{21}$

In conclusion our EAs of first-row hydrides, computed by FN-DMC simulations, whose largest standard deviations are $0.02 \mathrm{eV}$ for $\mathrm{NH}$ and $\mathrm{OH}$, differ from the experimental data by less than a standard deviation. We stress that these results have been obtained using standard quantum Monte Carlo calculations with fairly simple trial wave functions. The overall agreement between the experimental and the calculated data shows that cancellation of nodal errors has been achieved. On the whole this agreement is better than the one
TABLE III. Dissociation energies $D_{e}$ (in $\mathrm{kcal} \mathrm{mol}^{-1}$ ) calculated with the FN-DMC method compared with experimental results. Numbers in parentheses are one standard deviation in the last digit.

\begin{tabular}{lcc}
\hline \hline & Experiment $^{\mathrm{a}}$ & FN-DMC \\
\hline $\mathrm{LiH}^{-}$ & $48.26 \pm 0.48^{\mathrm{b}}$ & $48.39(7)$ \\
$\mathrm{BeH}^{-}$ & $47.02 \pm 2.31$ & $48.54(28)$ \\
$\mathrm{BH}^{-}$ & & $69.81(26)$ \\
$\mathrm{CH}^{-}$ & $82.85 \pm 2.31$ & $81.64(20)$ \\
$\mathrm{NH}^{-}$ & $74.88 \pm 3.46^{\mathrm{c}}$ & $71.63(44)$ \\
$\mathrm{OH}^{-}$ & $115.38 \pm 0.23$ & $116.15(42)$ \\
\hline \hline
\end{tabular}

${ }^{\mathrm{a}}$ Reference 17.

${ }^{\mathrm{b}}$ Reference 18.

${ }^{\mathrm{c}}$ Value corrected for the difference of about $18.45 \mathrm{kcal} \mathrm{mol}^{-1}$ between $\mathrm{N}+\mathrm{H}^{-}$products and $\mathrm{N}^{-}+\mathrm{H}$ (see Table III in Ref. 17).

achieved by previous $a b$ initio calculations. Better accuracy in the experimental data would be welcomed to provide a more stringent test of our results.

\section{ACKNOWLEDGMENTS}

Financial support by Università degli Studi di Milano is gratefully acknowledged. CPU time for this work was partially funded by the Centro CNR per Lo Studio delle Relazioni tra Struttura e Reattività Chimica, Milano.

${ }^{1}$ J. A. Pople, P. v. R. Schleyer, J. Kaneti, and G. W. Spitznagel, Chem. Phys. Lett. 145, 359 (1988).

${ }^{2}$ W. Kutzelnigg, Theor. Chim. Acta 68, 445 (1985).

${ }^{3}$ W. Klopper and W. Kutzelnigg, Chem. Phys. Lett. 134, 17 (1987).

${ }^{4}$ W. Kutzelnigg and W. Klopper, J. Chem. Phys. 94, 1985 (1991).

${ }^{5}$ R. J. Gdanitz, J. Chem. Phys. 110, 706 (1999).

${ }^{6}$ G. S. Tschumper and H. F. Schaefer, J. Chem. Phys. 107, 2529 (1997).

${ }^{7}$ B. L. Hammond, W. A. Lester, Jr., and P. J. Reynolds, Monte Carlo Methods in Ab Initio Quantum Chemistry, 1st ed. (World Scientific, Singapore, 1994).

${ }^{8}$ J. B. Anderson, in Quantum Mechanical Electronic Structure Calculations with Chemical Accuracy, edited by S. R. Langhoff (Kluwer, Dordrecht, 1995), p. 1.

${ }^{9}$ J. B. Anderson, Int. Rev. Phys. Chem. 14, 85 (1995).

${ }^{10}$ A. Luchow and J. B. Anderson, J. Chem. Phys. 105, 7573 (1996).

${ }^{11}$ R. N. Barnett, P. J. Reynolds, and W. A. Lester, Jr., J. Chem. Phys. 84, 4992 (1986).

${ }^{12}$ J. W. Moskowitz and K. E. Schmidt, J. Chem. Phys. 97, 3382 (1992).

${ }^{13}$ D. Bressanini, M. Mella, and G. Morosi, J. Chem. Phys. 108, 4756 (1998).

${ }^{14}$ P. E. Cade and Huo, J. Chem. Phys. 47, 614 (1967).

${ }^{15}$ K. E. Schmidt and J. W. Moskowitz, J. Chem. Phys. 93, 4172 (1990).

${ }^{16}$ C. J. Umrigar, K. G. Wilson, and J. W. Wilkins, Phys. Rev. Lett. 60, 1719 (1988).

${ }^{17}$ P. Rosmus and W. Meyer, J. Chem. Phys. 69, 2745 (1978).

${ }^{18}$ H. W. Sarkas, J. H. Hendricks, S. T. Arnold, and K. H. Bowen, J. Chem. Phys. 100, 1884 (1994).

${ }^{19}$ W. C. Stwalley and W. T. Zemke, J. Phys. Chem. Ref. Data 22, 87 (1993).

${ }^{20}$ G. L. Gutsev, M. Nooijen, and R. J. Bartlett, Phys. Rev. A 57, 1646 (1998).

${ }^{21}$ G. Frenking and W. Koch, J. Chem. Phys. 84, 3224 (1986).

${ }^{22}$ R. Rackwitz, D. Feldman, H. J. Kaiser, and E. Heinicke, Z. Naturforsch. Teil A 32, 594 (1977).

${ }^{23}$ A. Kasdan, E. Herbst, and W. C. Lineberger, Chem. Phys. Lett. 31, 78 (1975).

${ }^{24}$ D. M. Neumark, K. R. Lykke, T. Andersen, and W. C. Lineberger, J. Chem. Phys. 83, 4364 (1985).

${ }^{25}$ P. M. Schulz, R. D. Mead, P. L. Jones, and W. C. Lineberger, J. Chem. Phys. 77, 1153 (1982). 